\title{
Optical absorption in amorphous hydrogenated silicon nitride thin films deposited by the electron cyclotron resonance plasma method and subjected to rapid thermal annealing
}

\author{
F.L. Martínez ${ }^{a}$, I. Mártil ${ }^{a, *}$, G. González-Díaz , A.M. Bernal-Olivab , J.M. González-Leal ${ }^{\text {b }}$ \\ E. Márquez.
}

'Departanento de Fisica Aplicada HI, Universidad Complutense de Madrid, Madrid, Spain

'Departancho de Física de la Materia Condensada. Unversidad de Cádia Pueno Real. Spah

\begin{abstract}
We have analyzed the influence of rapid thermal annealing (from 300 to $1050^{\circ} \mathrm{C}$ ) on the optical properties of a- $\mathrm{SiN}_{x}: \mathrm{H}$. Three compositions were investigated: $x=0.97, x=1.43$ and $x=1.55$. Two different behaviors are observed depending on whether the as-grown nitrogen to silicon ratio of the samples is above or below the percolation threstold $(x=1.1)$ of Si-Si bonds, in the matrix of silicon ritride. The samples with $x=1.43$ and 1.55 experience an increase of the Tauc coeffient $(B)$ and a decrease of the Urbach parameter $\left(E_{0}\right)$ at low annealing temperatures, while at ligh temperatures the trend is inverted. On the contrary, the samples with $x=0.97$ show a slight and continuous increase of $B$ and a similar decrease of $E_{i j}$. The different behavior of the films with $x<1.1$ is explained by the percolation of the Si-Si bonds, which maintains the order of the structure at high annealing temperatures, preventing the inversion of the trends of $B$ and $E_{0}$. (C) 1999 Elsevier Science S.A. All rights reserved.
\end{abstract}

Kewwords: Silicon nitride; Optical properties; Electron cyclotron resonance method; Rapid thennal annealing

\section{Introduction}

$\mathrm{SiN}_{x}: \mathrm{H}$ thin films deposited by plasma techniques have found applications in different microelectronic devices such as silicon complementary metal-oxide-semiconductor (SiCMOS) devices and thin film transistors (TFTs). Each of these two applications requires different processing. For use in Si-CMOS transistors the plasma nitrides generally require post deposition annealing in an inert ambient. The combination of rapid thermal annealing (RTA) of silicon nitride films prepared by remote plasma enhanced chemical vapor deposition (RPECVD) was first introduced by the North Carolina State University group [1,2] and subsequenty discussed for ultra thin gate dielectrics [3,4]. For the thin film transistor application films must be defect free on deposition. This has also been discussed by the same group [5], as well as the bonding considerations for ideal performance of plasma nitrides [6]. The electron cyclotron resonance (ECR) plasma method shares the advantages of the remote plasma system and has also been used to produce

\footnotetext{
* Corresponding author. Tel.: + 34-913-944435; fax: + 34-913945196.

E-mail address: imartil@eucmax.sim,ucmes (1. Märti)
}

high quality silicon nitride thin films [7]. In a previous paper we have analyzed the physical processes that occur in the silicon nitride when it is subjected to RTA [8], but in this paper we put the main emphasis on the optical properties.

\section{Experimental}

We deposited thin films of a-SiN $: \mathrm{H}$ by ECR with an Astex AX4500 reactor. The precursor gases were $N_{2}$ and $\mathrm{SiH}_{4}$, and its flow ratio was varied to obtain films of three different compositions: $x=0.97,1.43$ and 1.55 (where $x$ is the ratio of nitrogen to silicon in the film). Films were grown without intentional heating on silica substrates. After deposition they were subjected to rapid thermal annealing in argon flow at temperatures ranging from 300 to $1050^{\circ} \mathrm{C}$ during $30 \mathrm{~s}$.

The optical analysis was performed using two different spectrometers and two complementary calculation methods. With the first method we measured the transmitance and reflectance spectra with a Perkin-Elmer Lambda 9 UVVIS-NIR spectrometer and calculated the optical constants following the procedure of Rer. [9]. In the secund case we 


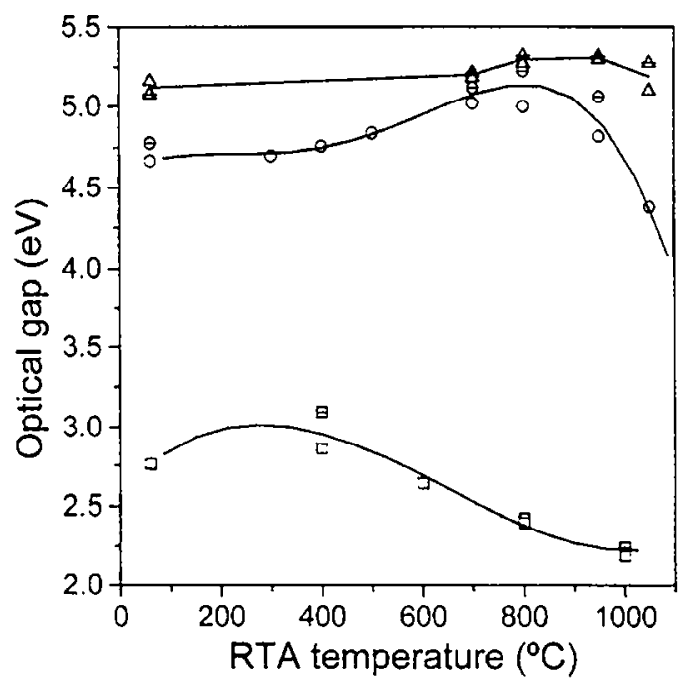

Fig. 1. Optical gap as a function of annealing temperature for the $\mathrm{SiN}_{r}: \mathrm{H}$ films with the three different compositions analyzed in this study. The values are obtained with the two different methods of calculation discussed in the text. The results with the method of Ref. [9] correspond to the asgrown compositions: $\sqsupset x=0.97 .0 x=1.43$ and $\Lambda x=1.55$, while the method of Ref. $[10]$ yields the results represented by: $\exists x=0.97$. $९ x=$ 1.43 and $\Delta x=1.55$. Lines are drawn as a guide for the eye.

measured only transmitance spectra with a Perkin-Elmer Lambda 19 spectrometer and the calculations were made following Ref. [10].

In the analysis of the results we will be concerned with the following three parameters: optical gap $\left(E_{i}\right)$, Tauc coefficient $(B)$ and Lrbach energy $\left(E_{j}\right)$. The first two are obtained by fitting the absorption coefficient to the well known Tauc law [11]

$\sqrt{\alpha h \nu}=B\left(h \nu-E_{\mathrm{g}}\right)$

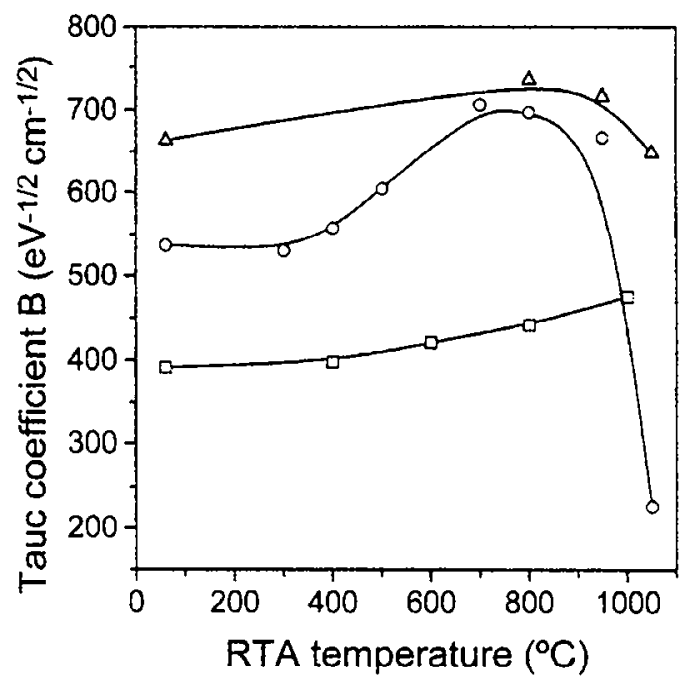

Fig. 2. Tauc coefficient versus annealing temperature. The values shown are the average of the values obtained with the wo methods analyzed in the lext and the as-grown compositions of the films are: $\sqsupset x=0.97 . \bigcirc x=$ 1.43 and $\Delta x=1.55$. Lines, are a guide for the eye.

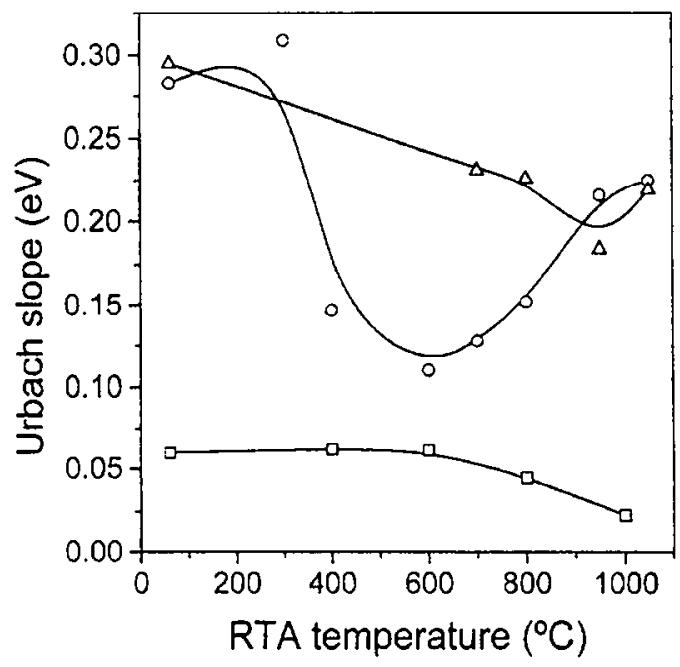

Fig. 3. Change of the Lrbach energy depending on the annealing temperawre for the three different as-grown compositions of the films: $\sqsubset x=0.97$. $O x=1.43$ and $\Delta x=1.55$. These values are vblained only with the anethod of Ref. [9]. Lines are a guide for the eye.

while the last one is obtained by fitting the values of $\alpha$ in the low absorption region to the equation [12]

$\alpha(\nu)=\alpha_{1} e^{h_{12} L_{i n}}$

\section{Results}

The values of the optical gap for the three different types of films are shown in Fig. 1. The films with as-grown $x=$ $1 .+6$ show a significant increase of the optical gap for moderate annealing temperatures, being the point of inversion of the trend between 700 and $800^{\circ} \mathrm{C}$. The other two types of film show much less pronounced variations and the turning point is at a lower temperature for the case of $x=0.97$ and at a higher one for $x=1.55$. We know from previous measurements of infrared spectra [8] of these films that those with as-grown $x=1.46$ show both $\mathrm{N}-\mathrm{H}$ and $\mathrm{Si}-\mathrm{H}$ bonds. the former predominating over the latter. In the case of the films with as-grown $x=0.97$ the $\mathrm{Si}-\mathrm{H}$ bonds predominate over the $\mathrm{N}-\mathrm{H}$ bonds. while in those with $x=1.55$ only $\mathrm{N}-\mathrm{H}$ bonds are observed. We also know from the measurements of composition [8] that the films with both $\mathrm{Si}-\mathrm{H}$ and $\mathrm{N}-\mathrm{H}$ bonds experience a loss of nitrogen for temperatures above $600^{\circ} \mathrm{C}$. while those without detectable $\mathrm{Si}-\mathrm{H}$ bonds $(x=1.55)$ maintuin a constant composition on annealing up to $1050^{\circ} \mathrm{C}$.

The values of the Tauc coefficient are plotted in Fig. 2 and those of the Urbach energy in Fig. 3. In the case of $B$, each data point is the average of the values obtained with the two methods. while for $E_{11}$ we only show the results from the method based on the transmission and reffection spectra because the method based only on the transmission spectrum does not yield values of the absorption coefficient deep enough into the Urbach edge. The inverse correlation exist 
ing between the trends of these two parameters had been observed before [13], but to our knowledge, not as a function of annealing temperature.

As it happened with the optical gap, it is the sample with as-grown $x=1.43$ that undergoes the larger variations in $B$ and $E_{0}$. This sample series has a maximum of the Tauc coefficient at about $700^{\circ} \mathrm{C}$ and a minimum of the Urbach energy around $600^{\circ} \mathrm{C}$. The films with as-grown $x=1.55$ do not have as noticeable a maximum and minimum, but still there is a slight increase of $B$ up to a turning point between 800 and $900^{\circ} \mathrm{C}$ and a minimum of $E_{0}$ at about $950^{\circ} \mathrm{C}$. The case of the series with as-grown $x=0.97$ is different. No maxima nor minima are observed. The values of the Tauc and Urbach parameters are roughly constant up to a temperature of $600^{\circ} \mathrm{C}$. At about this tempcraturc $B$ initiates a slight increase accompanied by a corresponding decrease in $E_{0}$.

\section{Discussion}

We will explain the results on the basis of the band structure of silicon nitride and the important concept of percolation of Si-Si bond chains in the amorphous lattice of silicon nitride. We know from our previous study [8] of the infrared spectra that there is a significant amount of non-bonded hydrogen in the films with $x=0.97$ and $x=1.43$. At moderate annealing temperatures this hydrogen forms bonds with $\mathrm{Si}$ and $\mathrm{N}$, especially with the first one, a fact that is observed in an increase of the $\mathrm{Si}-\mathrm{H}$ and $\mathrm{N}-\mathrm{H}$ bond density. The density of $\mathrm{Si}-\mathrm{H}$ bonds continues to increase with annealing temperature up to $500^{\circ} \mathrm{C}$ for the case of $x=$ 0.97 and up to $700^{\circ} \mathrm{C}$ for $x=1.46$, while the density of $\mathrm{N}-\mathrm{H}$ bonds starts to decrease at lower temperatures, indicating that the following well known network reaction is taking place [14]

$\mathrm{Si}-\mathrm{Si}+\mathrm{N}-\mathrm{H} \rightarrow \mathrm{Si}-\mathrm{N}+\mathrm{Si}-\mathrm{H}$

This reaction is based on the tendency for chemical ordering. A consequence of replacing $\mathrm{Si}-\mathrm{Si}$ bonds with $\mathrm{Si}-\mathrm{H}$ is that the valence band edge decreases and hence the optical gap increases. As the samples with $x=1.43$ have much more $\mathrm{N}-\mathrm{H}$ bonds than those with $x=0.97$, this reaction occurs to a much larger extent in the former than in the latter, explaining why the increase of the optical gap is higher in the films with $x=1.43$. In the films with $x=$ 0.97 the amount of $\mathrm{N}-\mathrm{H}$ bonds is not enough to produce a significant increase of the optical gap with annealing temperature. The case of the flims with $x=1.55$ is different in the sense that no $\mathrm{Si}-\mathrm{H}$ bonds are detected at any annealing temperature and no variation in the $\mathrm{N}-\mathrm{H}$ bond density is detected up to $900^{\circ} \mathrm{C}$. Therefore the above reaction is not taking place and the consequence is that there is no significant increase of the optical gap.

For temperatures above a certain threshold which depends on the composition the optical gap undergoes a decrease associated with the release of hydrogen and nitrogen. In the case of the films with both $\mathrm{Si}-\mathrm{H}$ and $\mathrm{N}-\mathrm{H}$ bonds this is explained by the following bond reaction

$\mathrm{Si}-\mathrm{H}+\mathrm{H}-\mathrm{SiN}-\mathrm{H} \rightarrow \mathrm{Si}-\mathrm{Si}+\mathrm{NH}_{3} \uparrow$

while for the films without $\mathrm{Si}-\mathrm{H}$ bonds the reaction is

$\mathrm{Si}-\mathrm{N}-\mathrm{H}+\mathrm{Si}-\mathrm{N}-\mathrm{H} \rightarrow 2(\mathrm{Si}-\mathrm{N})+\mathrm{H}_{2} \uparrow$

In the first case the destruction of $\mathrm{Si}-\mathrm{H}$ bonds and the formation of $\mathrm{Si}-\mathrm{Si}$ bonds will lower the gap, while in the second case it is well known that a large proportion of $\mathrm{N}-\mathrm{H}$ groups raises the conduction band edge leaving unaltered the valence band, and therefore it is expected a reduction in the optical gap when they are lost above $900^{\circ} \mathrm{C}$.

To explain the trends observed for the Taue coefficient and the Urbach energy we must resort to the concept of percolation. Both parameters are indicative of the amount of disorder in the network. When the disorder increases the Tauc coefficient decreases and the Urbach energy rises [13]. In Figs. 2 and 3 we have observed two different behaviors depending on whether the as-grown composition is above or below the percolation threshold $x=1$.1. Below $x=1.1$ there is a percolation of the $\mathrm{Si}-\mathrm{Si}$ bonds in the lattice and it is known that the $\mathrm{sp}^{3}$ hybridization of the silicon atoms form a tetrahedron which is a very rigid structure. When the silicon proportion is increased so that the stoichiometry $x$ falls below the percolation threshold, the connectivity of the covalent graph is enhanced and the high coordination number of silicon increases the number of constraints present. This has the effect of producing a structure which is much more rigid and stressed. As a consequence, the films with $x=0.97$ remain almost unaffected by the thermal relaxation. In the case of the samples with $x=1.43$ and 1.55 this thermal relaxation is clearly seen at temperatures below $600-700^{\circ} \mathrm{C}$ for the first case and $800-950^{\circ} \mathrm{C}$ for the second as an increase of $B$ and a decrease of $E_{0}$, while at temperatures above this an increase of band tailing indicates the destruction of order in the structure. These trends for the disorder parameters are stronger for the film with $x=1.43$ than for the one with $x=1.55$. At low annealing tenperature this difference is a consequence of the bond Eq. (3), which takes place for the former but not for the latter and it is known to be a reaction of chemical ordering. At high annealing temperatures the reason is that the films with as-grown $x=1.43$ lose nitrogen by means of bond Eq. (4) approximating their composition to the percolation threshold $x=1.1$, and it is known that the clustering that occurs around this threshold produces a strong tailing of band edges [13]. In that range of temperature the films with $x=1.55$ experience Eq. (5) and do not lose nitrogen.

\section{Conclusions}

We have studied the behavior of the optical gap. Tauc edge and Urbach tail in a-SiN $\mathrm{N}_{x}: \mathrm{H}$ as a function of annealing 
temperature and we have related the changes observed with the evolution of the hydrogen content and the composition. There are two main factors determining the observed behavior: the composition below or above the percolation threshold and the hydrogen bonds. The percolation of $\mathrm{Si}-\mathrm{Si}$ bonds will determine whether the disorder of the structure and the tailing of the band edges will be more or less affected by the thermal annealing. A composition below the percolation threshold means a rigid structure whose order will be little influenced by the annealing, while a composition above the percolation threshold means that thermal relaxation will be possible at moderate temperatures. On the other hand. the hydrogen content determines which network bond reactions will be possible. These reactions are responsible for the changes observed in the optical gap.

\section{References}

[1] Y. Ma. T. Yasuda. G. Lucovsky, J. Vac. Sci. Technol. A 11 (1993) 952.
[2] Y. Ma, T. Yasuda, G. Lucorsky, J. Vac. Sci. Technol. B 11 (1993) 1533.

[3] X. Ma. T. Y'asuda. G. Lucorsky. Appl. Phys. Lett. 64 (1994) 2226.

[f] G Lucovsky, H. Nïmi, Y. Wu. C.R. Parker, J.R. Hauser, J. Vac. Sci. Technol. A 16 11998) 1721.

[5] S.S. He. M.J. Williams. D.J. Stephens. G. Lucovsky, J. Non-Cryst. Solids 731 (1993) $16+-166$.

[6] G. Lucousky, G. Lucousky, J.C. Phillips, J. Non-Cryst. Solids 227 (1998) 1221 .

[7] S. García. I. Martil. G. González-Díaz, E. Castán, S. Dueñas, M. Fernández. J. Appl. Phys. 83 (1998) 332.

[8] F.L. Martínez, I. Mártil, G. González-Diuz. B. Selle, I. Sieber, J. NonCryst. Solids 227 (1998) 523.

[9] J.L. Hernández-Rojas, M.L. Lucia, L. Mártil. G. González-Diaz, J. Santamaria. F. Sánchez-Quesada, Appl. Opt. 31 (1992) 1606.

[10] R. Swanepoel. J. Phys. E: Sci. Instrum. 16 11983) 1214.

[11] J. Tauc, R. Grigorovici, A. Vancu, Phys. Stat. Sol. 15 (1966) 627

[12] R. Urbach. Phys. Rer. 92 (1953) 1324.

[13] J. Robertson. Philos. Mag. B 69 (1994) 307.

[14] Z. Yin. F.W. Smith, Phys. Rev. B 43 (1991) 4507. 